\title{
Selective Information Use and Perseveration in the Search Behavior of Infants and Young Children
}

\author{
Catherine Sophian \\ Carnegie-Mellon University
}

AND

Henry M. Wellman

University of Michigan

\begin{abstract}
Two experiments examined the early development of selective information use in search. The first experiment tested 9- and 16-month-olds on a modification of Piaget's Stage IV object permanence task. It examined infants' use of information from previous experiences with an object (prior information) and from the most recent hiding (current information) to locate a hidden object. In the second

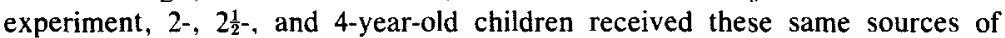
information along with new forms of prior and current information: information about the typical locations of objects (location specificity) and verbal information. No systematic perseveration was observed at 9 months, although previous findings related to perseveration were replicated. Perseveration was found at 16 months, but there was also evidence of selectivity at that age. When errors occurred, they tended to be to the prior location, but they were infrequent in comparison to correct searches at the current location. The preschoolers, while continuing to show perseveration, were more consistently selective than the infants. They also showed considerable generality in extending their selectivity to new sources of information.
\end{abstract}

The ability to locate objects that are not currently within view is one of the fundamental achievements of early cognitive development. In the first years of life, infants gradually develop the ability to search for objects where they have seen them hidden; their progressive achievements in

This research is based on a doctoral dissertation by the first author. It was supported by NICHHD Grant HD-13317 to the second author and by a grant from the Rackham School of Graduate Studies of the University of Michigan to the first author. We thank Mary Christiaanse for testing the infants in Experiment 1 and James Stigler for helping with data analysis. In addition, we are grateful to Carl Johnson, David Klahr, Karen Lind, and Robert Siegler for many useful suggestions for revising the manuscript. Send reprint requests to C. Sophian, Department of Psychology, Carnegie-Mellon University, Pittsburgh, PA 15213. 
this domain have been the focus of a substantial body of research on object permanence (Gratch, 1975; Harris, 1975; 1982; Piaget, 1954). Later, other sorts of information may be used to guide search as well, such as information about where an object was last used (Wellman, Somerville, \& Haake, 1979) or where it is typically located (Sophian \& Wellman, 1980).

In many search situations, more than one source of information may be available so that the child must select between them in determining where to search. Consider a 9-month-old in a typical object permanence task who finds a toy at one of two locations. Subsequently, the same toy is hidden in another location. There are, at a minimum, two sorts of information the infant could rely on to guide search. There is prior information-information about where the toy was hidden previouslyand there is current information-information about where the toy was hidden on this trial. The two sorts of information are in conflict since they indicate different search locations. Appropriately selective search would be based on current information in spite of the conflicting prior information. Infants of this age, however, have been characterized as relying on the prior rather than the current information, perseveratively searching at the initial location after the object was hidden in a new place (Piaget, 1954).

In contrast, consider an adult looking for her coat. She knows that her coat typically is hung in the closet, but her spouse tells her, "I just saw your coat in the car." Again two sources of potentially relevant information are in conflict. On problems like this, where information about an object's typical location conflicts with verbal information, even preschool children can be appropriately selective, searching on the basis of the more immediate verbal information (Sophian \& Wellman, 1980).

Comparing these two examples pinpoints three sorts of developments underlying the acquisition of selective search. First, the child must realize that selective information use is important. Presumably the simplest understanding here would involve an ability to select between current and prior information based on simple experience with the hiding and/or finding of the object. Second, hiding and finding information are not the only relevant sources of information. As depicted above, information about the typical locations of objects and verbal information also are relevant (as is information from maps, from the nature of the search space, etc.). Third, with the acquisition of an appreciation of some new source of information (e.g., verbal information), the searcher must integrate that information type into his or her selectivity considerations, learning what information it supercedes and is superceded by in conflict situations.

The present research examined the development of selective information use in infancy and early childhood. A major goal was to study infants, toddlers, and preschoolers in a common framework in order to facilitate understanding of the course of development from infancy through the 
preschool years. While the cognitive abilities of both infants and preschoolers have received considerable study in recent years, they have seldom been encompassed within a single investigation allowing direct comparisons between them. Partly this is because of the difficulty of finding tasks that can provide comparable, informative, findings across this developmentally divergent age range. Search tasks can serve this function.

In the present research we have used a search task to investigate the development of selective information use across five age groups: 9-month-

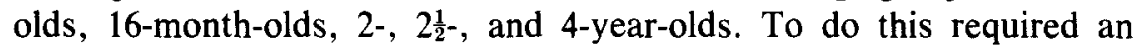
incremental method, because some kinds of information of interest (e.g., verbal information) could not be used with the youngest children. Hence, a core set of conditions was tested at all ages but others were added only for the older groups. Two experiments were conducted: one with 9- and 16-month-olds involving only combinations of hiding and finding

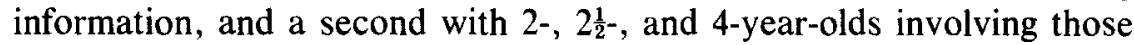
kinds of information along with two new ones-information about the typical locations of objects and verbal information. Both experiments included some sources of information which the youngest subjects might not yet be able to handle, in order to examine children's progress from incomprehension to appropriate use of the information. However, the incremental design minimized the presentation of conditions that were clearly beyond children's grasp while still tapping early phases in their use of new kinds of information.

\section{EXPERIMENT 1}

The first experiment was based on the Piagetian Stage IV object permanence task. In this, an object initially hidden at one location, A, is subsequently hidden at a new location, $B$. The classic finding has been that Stage IV infants continue to search at $\mathrm{A}$ even after watching the object being hidden at $\mathrm{B}$. There is little agreement, however, as to the causes of these perseverative errors.

In the present research, infants' search behavior in such situations was analyzed in terms of their use of alternative sources of information about an object's location. Two classes of information were varied. The first, prior information, pertained to an object's initial location. The second, current information, pertained to its location at the time of search. In order to determine whether and when children use these sources of information selectively it is necessary to have measures of three things: the child's use of current information when it alone is present, the child's use of prior information when it alone is present, and the child's information use when both sources of information are present in conflict situations. The design of the experiment was based on these three requirements. First there were A trials, which afforded 
a measure of infants' use of current information alone. Second, traditional B trials, which followed the A trials and involved hiding the object in a new place, represented the conflict condition in which both prior information (from the A trials) and current information (from the B hiding) was available. Finally, use of prior information alone was obtained by including some B trials on which the hiding was screened so that the infant would not see where the toy was hidden. Here the infant had no current information but could still rely on prior information from the preceding $\mathrm{A}$ trials.

Across problems, several different kinds of A trials were used in order to clarify what aspects of infants' experiences at A had the greatest impact on subsequent searches. Sometimes, the infant saw the object being hidden at $\mathrm{A}$ but was not allowed to find it there (hiding information only); sometimes, the infant did not see the object hidden at $A$ but was encouraged to search until s/he found it here (finding information only); and sometimes, as in the traditional Stage IV object permanence task, the infant both saw the object hidden at $\mathrm{A}$ and found it there (hiding and finding information).

\section{Method}

\section{Subjects}

Eighteen 9-month-olds (250 to 303 days; mean: 272 ) and 18 16-montholds ( 485 to 525 days; mean: 506 ) were tested. There were 11 boys and 7 girls in each age group. All infants lived in the Ann Arbor area and either were brought to a university laboratory or were tested at home.

\section{Materials}

Two sets of three $13 \times 7.5 \times 7-\mathrm{cm}$ plastic boxes, one blue and one orange, were used as hiding places. These boxes were open on the bottom and on one of the adjacent $13 \times 7-\mathrm{cm}$ sides (the back). Each set of boxes fit in a semi-circular arrangement on a $45 \times 29-\mathrm{cm}$ felt-lined tray, so that each box was about $35-\mathrm{cm}$ away from the infant. A screen fit into the tray behind the boxes. A set of small toys was used for target items.

\section{Procedure}

Infants were tested individually by a female experimenter. Testing began with three warm-up trials involving only a single location. First, a toy was partially hidden in the box and the infant was allowed to retrieve it. Second, the toy was hidden completely inside the box, and the infant again retrieved it. Finally, the toy was again hidden in the box, this time from behind the screen so that the infant could not see it being hidden, and once more the infant retrieved it. 
The experimental search problems were initiated using a different toy and the alternate set of boxes from those used for the warm-up trials. Throughout the experiment the boxes were exchanged for each search problem and a different toy was selected.

There were eight types of search problems, representing all combinations of four types of experience at A and two types of B hidings. The four types of experience at A consisted of (1) visible hiding - the experimenter hid the object at $A$ within view of the infant but then removed the object via the rear opening without allowing the infant to search for it; (2) finding experience-the experimenter hid the object at $\mathrm{A}$ from behind the screen and allowed the infant to find it (typically, infants had to search more than one location to do so, since initially they had no information about where to search); (3) one-trial hiding and finding-the experimenter hid the object at $\mathrm{A}$ within view of the infant and allowed him or her to find it; and (4) three trials hiding and finding - the experimenter hid the object at A within the infant's view and allowed him or her to find it, repeating the entire sequence three times. The two types of $B$ hidings consisted of either (1) a visible hiding-hiding the object within view of the infant; or (2) a screened hiding-hiding it from behind the screen. When the hiding was visible, the object was hidden in a location other than the one used for the preceding A experience. When it was screened, however, the object was hidden in the A location (thus an infant using prior information on those trials would be successful). On all problems, a third, control, location was present in addition to the A and $B$ locations. On all search trials, there was a 3-sec delay after the object was hidden before the infant was allowed to search.

Across search problems, different physical positions corresponded to the A location, the B location, and the control location (henceforth designated $\mathrm{C}$ ). The particular positions corresponding to these locations for each problem were varied across subjects according to a Latin square design.

If infants did not search correctly on the first $\mathrm{B}$ trial, they received a second B trial identical to the first. If they erred again, a third B trial was likewise administered.

Infants were tested on two blocks of search problems; each consisted of one problem of each of the eight types. Two different random orders were used for the problem types within each block. The order for the first vs second block was counterbalanced across subjects.

\section{Results}

Of primary interest was infants' performance on conflict trials, where they had both prior information (from the A trials) and current information (from watching the object hidden at B). Did infants select appropriately between these, relying on the current rather than the prior information, 
and hence perform correctly? Or did they rely on the prior rather than the current information and hence produce perseverative errors?

Several analyses were performed to characterize conflict trial performance, looking at both accuracy and error patterns. A clear evaluation of infants' selectivity, however, requires taking into account infants' performance on trials involving only prior or only current information as well as their performance on conflict trials. Analyses directed specifically to the issue of selectivity are presented in the second section of the results.

\section{Performance on Conflict Trials}

Correct responding. The mean proportion of conflict problems (i.e., visible-hiding $B$ trials) on which infants searched correctly at $B$ on the first B trial may be seen in Table 1. Nine-month-olds did not perform above chance (.33) in any of the conflict conditions, but 16-month-olds were well above chance in each condition. ${ }^{\prime}$ A 2 (age) $\times 2$ (sex) $\times 4$ (prior information) analysis of variance confirmed that older infants performed better than younger infants, $F(1,32)=46.94, p<.001$.

Error runs. When infants searched incorrectly on the first B trial, they were given up to two more trials in order to determine whether they would continue to make the same error. In previous studies, nonrandom strings of errors have constituted a major source of evidence that perseverative errors did not occur through merely random responding (e.g., Butterworth, 1975, 1976, 1977; Gratch \& Landers, 1971; Schuberth, Werner, \& Lipsitt, 1978). In the present study, when infants searched at $A$ on the first B trial, they continued to search there for an average of 1.80 trials at 9 months of age and 1.0 trials at 16 months. The former mean is marginally $(p=.06)$ greater than chance $(1.44)$; the latter is clearly below chance.

Similar nonrandom strings of errors were observed when infants' initial searches were at $\mathrm{C}$ rather than $\mathrm{A}$. The mean number of successive trials on which infants continued to search at $C$ was 2.04 at 9 months and 1.83 at 16 months. A 2 (age) $\times 2($ sex $) \times 2$ (error type) analysis of variance indicated no difference between the length of error runs to $A$ and to $C$, $F(1,14)=2.64, p>.10$, but confirmed that the error runs of 9-montholds were longer than those of 16 -month-olds, $F(1,14)=5.00, p<.05$.

\footnotetext{
All reported comparisons to chance are based on adjusted proportions which reflect performance only on those trials on which some response was made. This procedure was adopted because the occurrence of nonresponses alters the chance probabilities of particular responses somewhat. However, essentially the same results were obtained when tests were repeated using the overall proportions instead. All statements of significance are based on two-tailed $t$ tests with $p<.05$ or better.
} 
TABLE 1

Mean Proportion of Corkect Responses on Visible B Trials ${ }^{a}$ Experiment 1

\begin{tabular}{|c|c|c|c|c|}
\hline \multirow[b]{2}{*}{ Age } & \multicolumn{4}{|c|}{ A Experience } \\
\hline & $\begin{array}{c}\text { Visible } \\
\text { hiding at } \mathrm{A}\end{array}$ & $\begin{array}{l}\text { Finding } \\
\text { at } \mathrm{A}\end{array}$ & $\begin{array}{c}\text { One trial at } \\
\mathrm{A}\end{array}$ & $\begin{array}{c}\text { Three trials } \\
\text { at } \mathrm{A}\end{array}$ \\
\hline 9 months & $.42(.44)$ & .42 & $.31(.42)$ & .36 \\
\hline 16 months & $.86^{b}$ & $.92^{b}$ & $.83(.86)^{b}$ & $.75(.81)^{b}$ \\
\hline
\end{tabular}

Proportion of A errors. A direct test of the occurrence of nonrandom perseveration is afforded in the present study by the inclusion of the control location, C (Cummings \& Bjork, Note 1). If errors are random, they should be equally likely to be to $A$ and to $C$. If, however, they reflect systematic perseveration, they should be more often to $A$. The mean proportion of initial errors on conflict trials that were to $A$ rather than $\mathrm{C}$ was .52 at 9 months and .77 at 16 months. The former mean is not different from chance; the latter is significantly above chancc. A 2 (age) $\times 2$ (sex) analysis of variance confirmed that the proportion of errors to $\mathrm{A}$ was higher at 16 than at 9 months, $F(1,25)=4.73, \mathrm{p}<.05$.

\section{Selectivity}

In order to evaluate infants' selectivity in choosing between current and prior information, it is necessary to consider how they used each source of information separately as well as how they responded to conflicts between the two. Trials where only one source of information is available provide a baseline measure of the degree to which infants are sensitive to each kind of information, against which their use of the two kinds of information on conflict trials can be evaluated. Specifically, if infants do not use one source of information at all in their searches, even when it is the only information available, then it is not appropriate to speak of selectivity on conflict trials. On the other hand, if infants do use both kinds of information separately, then their selectivity on conflict trials can be evaluated by comparing their relative use of the two sources of information there to their relative use of the two when they were available separately.

Use of individual sources of information. Infants' use of current hiding information alone (in the absence of conflicting prior information) is reflected in their performance on the first $A$ trial of problems involving one or three trials at A. The mean proportion of correct searches on these trials was .56 at 9 months and .90 at 16 months of age; both are significantly above chance. Sixteen-month-olds performed significantly better than 9-month-olds, $F(1,34)=19.86, p<.001$. 
TABLE 2

Mean Proportion of Correct Responses on Screened B Trials ${ }^{a}$ Experiment 1

\begin{tabular}{lcccc}
\hline & \multicolumn{4}{c}{ A Experience } \\
\cline { 2 - 5 } Age & $\begin{array}{c}\text { Visible } \\
\text { hiding at A }\end{array}$ & $\begin{array}{c}\text { Finding at } \\
\mathrm{A}\end{array}$ & $\begin{array}{c}\text { One trial at } \\
\mathrm{A}\end{array}$ & $\begin{array}{c}\text { Three trials } \\
\text { at A }\end{array}$ \\
\hline 9 months & $.31(.41)$ & $.19(.21)$ & $.31(.33)$ & $.28(.38)$ \\
16 months & $.53(.56)^{b}$ & .39 & $.50(.56)^{b}$ & $.42(.50)$ \\
\hline
\end{tabular}

"Adjusted proportions, based on only those problems on which infants searched at least one location, are indicated in parentheses when they differ from the overall proportions.

${ }^{b}$ Significantly above chance (.33) at $p \leqslant .05$.

Use of prior information alone (in the absence of conflicting current information) is reflected in infants' performance on $B$ trials involving screened hidings. The mean proportion of correct searches (at $A$ ) on the first B trial of each of the problem types involving screened B trials may be seen in Table 2. Nine-month-olds did not use any form of prior information to a significant extent, whereas 16-month-olds did (see Table 2). When 16-month-olds' performance was averaged across the four prior information conditions, they clearly showed above chance use of the prior information. This seems the best way to consider the data since a 2 (age) $\times 2$ (sex) $\times 4$ (prior information) analysis of variance indicated no differences among the forms of prior information. The analysis of variance confirmed that 16-month-olds used the prior information more than 9 . month-olds did, $F(1,32)=8.57, p<.01$.

Selectivity ratios. A direct test of infants' selectivity in choosing between prior and current information on conflict trials was made by comparing infants' observed performance on those trials with an expected value based on their use of prior and current information separately. An observed selectivity ratio measured infants' relative use of prior and current information on conflict trials. A predicted selectivity ratio measured their relative use of the same kinds of information when they were available separately (on screened B trials and visible-hiding A trials). Infants who were appropriately selective in choosing between the competing sources of information should have relied less on the prior information and more on the current information on conflict trials than would be expected from their use of the two kinds of information separately; hence, comparisons between the two ratios provide a test of selective information use. ${ }^{2}$

An assumption underlying this analysis is that children in fact make some use of both sources of information. For 9-month-olds, there is no evidence of use of prior information on either screened or visible B trials.

2 Specifically, the observed selectivity ratio was

No. of initial searches at $A$ on conflict trials

No. of initial searches at $B$ on conflict trials 
For 16-month-olds, however, there is clear evidence for the use of both kinds of information. Therefore, selectivity ratios were examined only for 16-month-olds. Their observed ratios were significantly smaller than the predicted, .13 vs .50 (Scheffe test, $p=.05$ ), indicating appropriate selectivity on the conflict problems.

Second choices after errors to A. One further piece of information relevant to the issue of selectivity is infants' second choices on conflict trials, following initial errors to the A location. These second choices provide an indication of whether infants remembered the current information even when they searched at the prior location (Webb, Massar, \& Nadolny, 1972). If they did, then the errors clearly reflect a failure of selectivity since infants failed to use the current information that was available to them.

The mean proportion of those $\mathrm{B}$ trials involving an initial error to the A location on which infants searched the correct location as a second choice was .32 for 9 -month-olds and .80 for 16 -month-olds. The mean for 16-month-olds was above chance (.50); the one for 9-month-olds was not.

\section{Discussion}

Clear developmental improvements were found in infants' searches for an object about which they had conflicting information. On conflict trials, 16-month-olds were both more likely to search at the correct B location and less likely to search at the incorrect A location than 9month-olds were. They also more readily corrected the errors they did make, as the analyses of error runs indicate.

More informative than this general developmental trend are the patterns of information use evident in the searches of the two age groups. These patterns may be most clearly explicated by considering infants' search patterns in terms of perseveration and selectivity.

\section{Perseveration}

Nine-month-olds have been widely characterized as perseverative on the basis of previous work (Bremner, 1982; Gratch, 1975, 1977; Harris, 1975, 1982; Piaget, 1954). As we understand it, this characterization implies a systematic use of information derived from A trials as a basis for search on subsequent $B$ trials. The present results, however, while indicating considerable search at A on B trials, provide no evidence of a systematic use of prior information underlying those errors. In particular, the fact that 9-month-olds' errors on conflict trials were as likely to be

The predicted selectivity ratio was

$$
\text { No. of initial searches at A on screened B trials }
$$

The observed ratio should be less than the predicted if infants are appropriately selective. 
to the control location, $\mathrm{C}$, as to the prior location, A, suggests that their errors were not based on a systematic tendency to return to the prior location.

There are a number of differences between the procedures used in this study and those used in previous work that might have contributed to the failure to corroborate perseveration here. Notably, the use of three rather than two alternative locations, and the presentation of a lengthy series of problems to each infant, are potentially significant deviations from traditional testing procedures. Two considerations, however, suggest that the present findings have more general implications for infant perseveration than simply reflecting a methodological peculiarity. First, the results of the present study are in fact quite comparable to previous findings and suggest a different conclusion primarily because of the inclusion of controls that have not typically been available. Second, even the results of previous research provide indications that perseveration may be a more restricted phenomenon than traditional accounts suggest.

The present data replicate three phenomena noted in previous studies. First, on conflict trials 9-month-olds were about equally likely to search at $\mathrm{A}$ and at the correct $\mathrm{B}$ location, a distribution of searches found in many (c.g., Butterworth, 1975, 1977; Evans, Note 2; Evans \& Gratch, 1972) although not all (e.g., Bremner \& Bryant, 1977; Butterworth, 1976; Gratch \& Landers, 1971; Schuberth et al., 1978) previous studies. Second, the error patterns of 9-month-olds were not random but reflected considerable persistence of errors over trials (Butterworth, 1975, 1976, 1977; Evans \& Gratch, 1972; Gratch, Appel, Evans, LeCompte, \& Wright, 1974; Landers, 1971; Schuberth et al., 1978). And third, while the proportion of A (vs C) errors was greater for 16- than 9-month-olds, the absolute frequency of errors to A declined with age, as previous studies have found (Butterworth, 1976; Gratch \& Landers, 1971).

Evidence questioning the characterization of errors to $\mathrm{A}$ as perseverative comes from comparisons to the control location, C. Although errors to A were quite frequent at 9 months, they were not more frequent than errors to C. Moreover, error runs, which have been taken as evidence of nonrandom responding and hence of perseveration, were as long when the initial error was to $C$ as when it was to $A$ and hence do not appear to reflect a specific perseverative tendency. In sum, when considered in relation to searches at a control location, neither the simple occurrence of errors to A nor their persistence over successive trials appears to provide sufficient grounds for interpreting those errors as perseverative.

Nevertheless, it would bc unwise to conclude from the present findings that all previous reports of perseveration have been artifacts of a failure to include a control location. In particular, the reports of perseveration by a large majority of infants in some studies are difficult to account for in terms of random responding. A more tenable conclusion would appear to be that perseveration in fact occurs under some circumstances but is 
considerably more restricted than previously concluded. Certainly, the degree of perseveration observed has varied widely even within studies using the traditional two-location object permanence task, although the specific factors accounting for this variation are not yet well understood. Moreover, researchers have begun to identify some specific restrictions on the conditions under which perseveration obtains. For instance, in an influential study Gratch et al. (1974) showed that it is necessary to impose a delay between hiding and search in order to get perseveration. More recently, using a somewhat different task, Acredolo (1979) showed that infants will no longer make egocentric spatial responses if they are tested in the familiar surroundings of their own home, and Bremner (1978) showed that similar kinds of egocentric responding could be overcome by using highly differentiated locations as hiding places. These recent findings clearly dovetail with the present results in suggesting that egocentrism or perseveration is by no means a pervasive characteristic of infants' spatial behavior, although it may be elicited under some circumstances.

\section{Selectivity}

The question of selectivity has to do with how infants resolve conflicts between competing sources of information. Hence, it presupposes that at least two sources of information are being taken into account. Among the 9-month-olds, examination of selectivity was precluded by the fact that only one source of information-the current information-reliably influenced infants' searches; among the 16-month-olds, however, there was evidence of the use of both prior and current information, making examination of selectivity feasible. These infants did show selectivity in that they used current information more, relative to prior information, on conflict trials than would be expected from their use of the two kinds of information separately. At the same time, however, they made perseverative errors which reflect the use of prior rather than current information on at least some trials. Moreover, the accuracy of their second choices indicates that these errors were not restricted to trials on which the current information had been forgotten. In combination, these findings present a picture of significant, but still limited, selectivity at 16 months of age.

\section{EXPERIMENT 2}

The purpose of the second experiment was to examine further developments in selective information use in search, linking the early abilities manifested by the infants in Experiment 1 to continuing developments in search extending through the preschool years. Two issues were of particular interest. First, since 16-month-olds show reliable deviations from selectivity, it is important to chart further developments in making the same kinds of selections among older children. In several studies, 
continuing perseverative errors have been noted among preschoolers (e.g., Horn \& Myers, 1978; Loughlin \& Daehler, 1973; Sophian \& Wellman, 1980 ), raising the question of whether these children still have difficulty consistently making the appropriate selection between prior and current information or whether their perseverative errors have a different basis.

A second issue concerned the generality of developing skills for selective information use. While the first experiment focused exclusively on infants' use of information derived from hiding and finding events, other sources of information are clearly relevant at older ages. Two important ones are verbal information and information about the typical locations of objects.

The design of Experiment 2 was essentially an expansion of that of Experiment 1 . All of the conditions from that experiment were included, along with several new conditions which resulted from the introduction of a new form of prior information and a new form of current information.

The new form of prior information was derived from children's knowledge about the typical locations of objects (which we refer to as location specificity). On some trials, the $\mathrm{A}, \mathrm{B}$, and $\mathrm{C}$ locations were made to represent rooms of a house to which the target items were differentially associated. As with the other forms of prior information, these associations sometimes represented the only information available to the child about where to search and sometimes were in conflict with other, current, information. In view of the parallel between location specificity and experiences at $\mathrm{A}$ as sources of prior information in this study, it will be convenient to refer to the location indicated by location specificity information as $\mathrm{A}$ and the location indicated by any conflicting current information as $\mathbf{B}$.

The new form of current information consisted of verbal statements about an object's location. Sometimes, instead of allowing children to watch as an object was hidden on B trials, the object was hidden from the rear but its location was indicaled verbally. This new form of current information was presented in conjunction with each of the types of prior information.

\section{Method}

\section{Subjects}

Eighteen 2-year-olds ( $2 ; 1$ to $2 ; 5 ;$ mean: $2 ; 3)$, eighteen $2 \frac{1}{2}$-year-olds ( $2 ; 6$ to $2 ; 10$; mean: $2 ; 9)$, and eighteen 4 -year-olds ( $4 ; 0$ to $4 ; 7$; mean: $4 ; 4)$ were tested, with approximately equal numbers of boys and girls in each group. All children lived in the Ann Arbor area and were tested either at a university laboratory or at a nearby preschool.

\section{Materials}

Plastic boxes like those used in the infant study served as hiding places. White cards depicting cither animals (dog, bird, and cat; one per card) 
or rooms (bedroom, kitchen, and bathroom) could be placed on the front of the boxes for the various search problems. These cards made it possible to refer to the boxes with a verbal label (e.g., "the bedroom," "the dog's house"). The boxes again fit in a semicircle, with a screen behind them.

Six objects were used as targets, two associated with each of the rooms. The bedroom-specific objects were a pillow and a teddy bear; the bathroom-specific objects were a toothbrush and a bar of soap; and the kitchen-specific objects were a spoon and a box of crackers.

\section{Procedure}

Children were tested individually by a female experimenter. They were first asked a series of pretest questions to assess their knowledge of the verbal labels for the animals and rooms and of the location specificity relations between the target objects and the rooms. Next they received two warm-up trials to familiarize them with the search task; and finally, they received the experimental search problems.

For the pretest, the experimenter placed the three boxes on the tray with the animal cards on them. She named one of the animals and asked the child to point to it. This procedure was repcated for the sccond and then the third animal. The animal cards were then replaced with the room cards for the second part of the pretest. As with the animals, the experimenter named one room at a time and asked the child to point to it. For the final part of the pretest, the room cards remained in place. The experimenter presented the target objects to the child one at a time, asking the child to point to the room which "would be the best place for this."

For the warm-up trials, a single box was placed on the tray and an object was hidden within it. The first warm-up trial involved hiding a balloon in the box in front of the screen; the second involved hiding the balloon in the box again, but this time from behind the screen.

For the experimental search problems, all three boxes were placed on the tray. There were 15 types of problems, resulting from the factorial combination of five types of prior information with three types of current information. The types of prior information consisted of the four types of A experience from the infant study plus the location specificity information. Location specificity information was manipulated by varying whether the animal cards or the room cards were placed on the boxes, since the location specificity of the target objects was only relevant to the rooms. Thus, on problems involving location specificity information the three boxes were made to represent a kitchen, bedroom, and bathroom; on the remaining problems they were marked by pictures of a bird, a cat, and a dog. The three types of current information consisted of the two types of B hidings-visible and screened-from the infant study plus a verbal information condition. On verbal information trials, the object 
was hidden from behind the screen but the experimenter indicated verbally where it would be, saying, e.g., "The soap will be in the bird's box" or, "The soap will be in the bedroom." It should be noted that, as in the infant study, when current information was available on B trials it always pointed to a different location than the prior information did (resulting in a conflict trial), and when no current information was available the object was always hidden at $\mathrm{A}$.

The procedures for administering additional trials if children did not search correctly on the initial B trials were the same as in the infant study. Also, as in that study, children received two blocks of search problems, each containing one problem of each type, and problems within the two blocks were presented in different random orders, counterbalanced over subjects. Different physical positions again corresponded to the A, $\mathrm{B}$, and $\mathrm{C}$ locations on different problems. The positions corresponding to the $\mathrm{A}, \mathrm{B}$, and $\mathrm{C}$ locations for each problem were varied across subjects according to a Latin square design.

\section{Results}

Initial analyses examined children's performance on the pretest questions assessing their knowledge of the verbal labels for locations and of the location specificity relations between objects and locations. Subsequent analyses then focused on children's use of the various forms of current and prior information in their searches. In most respects these latter analyses paralleled those carried out on the data from the previous study, except for the inclusion of the new sources of information provided in this study.

\section{Pretest Performance}

Children's performance on pretest questions may be seen in Table 3 . Children at all three ages were above chance on the questions testing for knowledge of the verbal labels of animals, and of the verbal labels

TABLE 3

Proportion of Correct Responses to Pretest Questions ${ }^{u}$ Experiment 2

\begin{tabular}{lccc}
\hline & \multicolumn{3}{c}{ Question type } \\
\cline { 2 - 4 } Age & $\begin{array}{c}\text { Animal } \\
\text { labels }\end{array}$ & Room labels & $\begin{array}{c}\text { Location } \\
\text { specificity }\end{array}$ \\
\hline 2 years & $.93^{b}$ & $.57(.65)^{b}$ & $.34(.37)$ \\
2 $1 / 2$ years & $.93^{b}$ & $.87^{b}$ & $.90^{b}$ \\
4 years & $1.00^{b}$ & $1.00^{b}$ & $.99^{b}$ \\
\hline
\end{tabular}

a Adjusted proportions, based on only those questions to which some response was made, are indicated in parentheses when they differ from the standard proportions.

${ }^{b}$ Significantly above chance $(.33)$ at $p \leqslant .001$. 
for the rooms. However, only the older two groups were above chance on the location specificity questions.

There were significant age differences in performance on the pretest questions testing location specificity knowledge, $F(2,51)=67.50, p<$ .001 , and on those testing knowledge of the verbal labels for the rooms, $F(2,51)=10.39, p<.001$, but not on the questions assessing knowledge of the verbal labels for the animals, $F(2,51)=2.03, p>.10$. On both the location specificity measure and the measure of knowledge of the verbal labels for the rooms, the 2-year-olds did significantly less well than either of the older groups, who did not differ from each other (Scheffe tests, $p=.05$ ).

\section{Performance on Conflict Trials}

Correct responding. The mean proportion of conflict problems on which children searched correctly at B on the first B trial may be seen in Table 4. Performance was above chance in most conditions (see Table 4), indicating that children at all three ages were able to use both current hiding information and verbal information to guide their searches.

The mean proportion of correct responses in each of the conflict conditions was compared in a 3 (age) $\times 2$ (sex) $\times 2$ (current information) $\times$ 5 (prior information) analysis of variance. There were significant main effects of age, $F(2,48)=18.30, p<.001$, and current information, $F(1$, $48)=21.55, p<.001$, and interactions of age $\times$ current information, $F(2,48)=12.94, p<.002$, and current information $\times$ prior information, $F(4,192)=2.65, p<.05$. Overall, 2 -year-olds performed less well than either $2 \frac{1}{2}$-year-olds or 4 -year-olds, who did not differ from each other (Scheffe tests, $p=.05$ ). The age differences were only significant, however, when the current information was provided verbally rather than through a visible hiding. ${ }^{3}$ Likewise, while overall performance was better when current information was provided through a visible hiding than when it was provided verbally, this difference was only significant for 2-yearolds (Scheffe tests, $p=.05$ ).

Error runs. When children searched at A on the first B trial of conflict problems, they continued to search there for an average of 1.36 trials at 2 years, 1.13 trials at $2 \frac{1}{2}$ years, and 1.06 trials at 4 years of age. The mean for 2-year-olds did not differ from chance, but the other two means were below chance. When children searched at $\mathrm{C}$ on the first $\mathrm{B}$ trial, they continued to search there for an average of 1.6 trials at 2 years and 1.0 trials at each of the other two ages. A 3 (age) $\times 2$ (sex) $\times 2$ (error type) analysis of variance indicated a significant decrease in run lengths with age, $F(2,5)=6.29, p<.05$, but no difference between error runs to $\mathrm{A}$ and those to $\mathrm{C}, F(1,5)=1.23, p>.10$.

${ }^{3}$ All age differences pertaining to children's use of verbal or location specificity information in search remained significant in analyses of covariance controlling for the relevant knowledge, as assessed by the pretest. 
TABLE 4

mean Proportion of Correct Responses in Conditions Involving Conflicting Prior

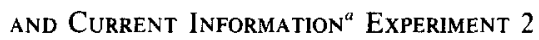

\begin{tabular}{|c|c|c|c|c|c|}
\hline \multirow[b]{2}{*}{$\begin{array}{l}\text { Current } \\
\text { information }\end{array}$} & \multicolumn{5}{|c|}{ Prior information } \\
\hline & $\begin{array}{c}\text { Visible } \\
\text { hiding } \\
\text { at } A\end{array}$ & $\begin{array}{c}\text { Finding } \\
\text { at } \mathrm{A}\end{array}$ & $\begin{array}{l}\text { One } \\
\text { trial at } \\
\text { A }\end{array}$ & $\begin{array}{l}\text { Three } \\
\text { trials } \\
\text { at } A\end{array}$ & $\begin{array}{c}\text { Location } \\
\text { specificity }\end{array}$ \\
\hline \multicolumn{6}{|c|}{ Verbal statement } \\
\hline 2 years & $.53^{b}$ & $.53^{b}$ & $.56^{b}$ & .53 & $.47(.50)$ \\
\hline $21 / 2$ years & $.82^{\prime}$ & $.81^{\prime}$ & $.78^{\prime}$ & $.83^{c}$ & $.78^{\circ}$ \\
\hline 4 years & $.89^{c}$ & .97 & $.92^{c}$ & $.94^{c}$ & $.92^{c}$ \\
\hline \multicolumn{6}{|c|}{ Visible hiding } \\
\hline 2 years & $.92^{c}$ & .92 & $.78^{\circ}$ & $.72^{\circ}$ & $.97^{\circ}$ \\
\hline $21 / 2$ years & $.94^{c}$ & .94 & $.81^{\circ}$ & $.92^{c}$ & $.86^{\circ}$ \\
\hline 4 years & $.89^{c}$ & .97 & $.86^{\prime}$ & $.83^{\prime}$ & $.97^{\circ}$ \\
\hline
\end{tabular}

" Adjusted proportions, based on only those problems on which children searched at least onc location, are indicated in parentheses when they differ from the overall proportions.

${ }^{b}$ Significantly above chance $(.33)$ at $p \leqslant .05$.

c Significantly above chance $(.33)$ at $p \leqslant .001$.

Proportion of A errors. The proportion of errors to A rather than C on the first B trial of conflict problems averaged .69 at 2 years, .57 at $2 \frac{1}{2}$ years, and .77 at 4 years of age. The means for the 2-year-olds and the 4-year-olds were significantly above chance, but that for the $2 \frac{1}{2}$-yearolds was not. However, age differences were not significant in a 3(age) $\times 2($ sex) analysis of variance, $F(2,37)=1.67, p>.10$. When all three age groups of preschoolers were combined, the proportion of $A$ errors was significantly above chance.

\section{Selectivity}

Use of individual sources of information. Children's use of current hiding information by itself is reflected in their performance on the first A trials of problems involving one or three trials at $\mathrm{A}$. The mean proportion of correct searches on those trials was .93 at each of the three ages; all were significantly above chance.

Since verbal information was only provided on B trials, it was never available without some form of conflicting prior information also being present. Children's use of the verbal information on these trials may therefore underestimate their ability to make use of verbal information in search. Nevertheless, children responded in accordance with the verbal information on .53 of the problems at 2 years, on .81 of the problems at $2 \frac{1}{2}$ years, and on .93 of the problems at 4 years of age. All three groups were above chance, but there were significant differences between them, $F(2,51)=22.59, p<.001$. Two-year-olds searched in accordance with 
the verbal information less often than either of the older groups, who did not differ from each other (Scheffe tests, $p=.05$ ).

Children's use of prior information by itself is reflected in their performance on screened B trials. The mean proportion of correct responses as a function of the kind of prior information available may be seen in Table 5. Performance in the four conditions involving prior information from A trials was well above chance, but only 4-year-olds used location specificity significantly.

A 3(age) $\times 2$ (sex) $\times 5$ (prior information) analysis of variance indicated significant main effects of age, $F(2,48)=3.66, p<.05$, and prior information, $F(4,192)=13.04, p<.001$, and an interaction between them, $F(8,192)=2.63, p<.01$. Overall, 4-year-olds performed better than 2-year-olds, and performance was worse when the prior information consisted of location specificity than in other conditions. However, differences among the conditions were significant only at the younger two age levels, and age differences were significant only when the prior information consisted of either finding experience at $\mathrm{A}$ or location specificity (Scheffe tests, $p=.05$ ).

Selectivity on conflict trials. Several sets of analyses examined children's selectivity when faced with different kinds of conflicting information. First, children's selectivity between prior information from A trials and current hiding information-the kind of selectivity examined for 16month-olds in Experiment 1-was examined. Note that all three groups in the present study showed use of both these classes of information separately, so this selectivity analysis could be carried out at each age level. Second, children's selectivity between location specificity and current hiding information was examined. Here, since the 4-year-olds were the only group to show significant use of location specificity alone, they were

TABLE 5

Mean Proportion of Correct Responses in Conditions Involving only Prior INFORMATION" EXPERIMENT 2

\begin{tabular}{|c|c|c|c|c|c|}
\hline \multirow[b]{2}{*}{ Age } & \multicolumn{5}{|c|}{ Prior information } \\
\hline & $\begin{array}{c}\text { Visible } \\
\text { hiding at } \\
\mathrm{A}\end{array}$ & $\begin{array}{l}\text { Finding } \\
\text { at } \mathrm{A}\end{array}$ & One trial at $\mathrm{A}$ & $\begin{array}{c}\text { Three } \\
\text { trials at } \\
\text { A }\end{array}$ & $\begin{array}{l}\text { Location } \\
\text { specificity }\end{array}$ \\
\hline 2 years & $.69^{\circ}$ & .36 & $.67(.69)^{6}$ & $.89^{\prime}$ & .19 \\
\hline $21 / 2$ years & $.72^{x}$ & $.53^{b}$ & .72 & $.67^{\prime}$ & .39 \\
\hline 4 years & $.72^{\mathrm{c}}$ & $.69^{\circ}$ & $.69^{\prime}$ & $.81^{\circ}$ & $.78^{\circ}$ \\
\hline
\end{tabular}

a Adjusted proportions based on only those problems on which children searched at least one location, are indicated in parentheses when they differ from the overall proportions.

${ }^{b}$ Significantly above chance (.33) at $p \leqslant .05$.

c Significantly above chance (.33) at $p \leqslant .001$. 
the only ones for whom this selectivity analysis was performed. Finally, children's selectivity in using verbal information was of interest. In this case, selectivity ratios could not be computed because verbal information was never presented alone. Nevertheless, the data from other conditions present a clear picture of selectivity.

Consider first the selectivity ratios for conflicts between prior information from A trials and current hiding information. Predicted and observed ratios, calculated just as in Experiment 1, averaged .71 and .18 at 2 years, .72 and .08 at $2 \frac{1}{2}$ years, and .79 and .14 at 4 years of age. All three groups showed selectivity on conflict trials, in that they used the current information more, relative to the prior information, than would be expected from their use of the two separately, $F(1,48)=203.97, p$ $<.001$. The difference between the two ratios did not differ across age groups, $F<1$.

Next consider 4-year-olds' selectivity between location specificity and current hiding information. Again, predicted and observed ratios were used to evaluate this form of selectivity. The predicted ratio was the ratio of use of location specificity alone to use of current hiding information alone; it averaged .72. The observed ratio was the ratio of the same two kinds of responses on trials on which the two kinds of information were in conflict; it averaged .06. Comparison of the two ratios again indicated selectivity: use of current hiding information on conflict trials was significantly greater than would be expected from performance when that information and location specificity were available separately, $t(17)=$ $5.21, p<.001$.

The issue of children's selectivity in using verbal information can again be subdivided into the subissues of whether children are selective in cases of conflicts with prior information from A trials, and whether they are selective in cases of conflicts with location specificity.

In the first case, the relevant findings are that all three groups of children used the prior information from A trials when it was the only information available (see "Use of individual sources of information" above), yet on conflict trials they responded in accordance with the verbal information 54,81 , and $93 \%$ of the time at $2,2 \frac{1}{2}$, and 4 years (see "Performance on conflict trials: correct responding"). Hence it is clear that children were able to use the prior information but relied on the verbal information instead when it was available.

In the case of conflicts between verbal and location specificity information, the question of selectivity again applies only to the 4-year-olds, since they were the only ones who used location specificity information by itself. When location specificity conflicted with verbal information, these children used the verbal information $92 \%$ of the time, once more presenting a clear picture of selectivity.

Second choices after errors to $A$. The mean proportion of initial searches 
at $\mathrm{A}$ on conflict trials that were followed by a second search at the correct location was again examined for indications of whether children remembered the current information when they went to the prior location. This proportion averaged .49 for 2 -year-olds, .48 for $2 \frac{1}{2}$-year-olds, and .60 for 4 -year-olds. None of these proportions differed from chance.

\section{Discussion}

All three of the age groups in this experiment, like the 16-month-olds in Experiment 1, were selective in that they generally relied on current rather than prior information when the two were in conflict. Perseverative responses continued to account for the majority of the errors that occurred, but these errors were infrequent; specifically, they occurred less frequently than would be expected from children's use of prior and current information separately.

One kind of selectivity evidenced by the children in this study involved relying on current hiding information over prior information from $\mathrm{A}$ trials-a form of selectivity that was already evident among 16-montholds in Experiment 1. Preschoolers showed an advance over 16-montholds, however, in that they were more consistently selective and no longer tended to search the correct location as a second choice following errors to A. Hence, there was no evidence that they remembered the current information when they searched at the prior location. Rather than reflecting lapses from appropriate selectivity, their perseverative responses appear to represent occasional instances in which children had forgotten the current information and so turned to the prior information as the only cue available, just as they did on screened-hiding B trials.

Preschoolers also showed considerable generality in their selective information use. The kind of conflict to which 16-month-olds had responded selectively-involving current vs prior forms of hiding and finding information-was only one of several situations in which preschoolers showed selectivity. In fact, preschoolers responded selectively to all of the kinds of conflicts on which they were tested, showing appropriate use of verbal and location specificity information as well as of the simpler hiding and finding information.

First, preschoolers selectively used current hiding information over location specificity as a basis for search, as attested to by the analysis of selectivity ratios. This analysis could only be performed with 4-yearolds, since the younger groups did not use location specificity information at all in their searches. The important point, though, is once children had begun using the location specificity information, they were able to select appropriately between it and competing information.

Second, children were selective in relying on current verbal information over prior information from A trials. At all three ages, children were able to use verbal information correctly to guide search on B trials, in 
spite of conflicting prior information from A trials. Since other analyses (e.g., use of A information on screened B trials) indicated that preschoolers did remember A information into B trials, it seems clear that current verbal information was used selectively (in preference to prior hiding information) to guide search. This conclusion receives further support from another study, which demonstrated appropriate selectivity between these two kinds of information with children as young as 18 months of age (Sophian, 1982).

Finally, the present data show selective use of current verbal information over location specificity information among the 4-year-olds, who were the only group to use location specificity in their search. Again, they performed correctly on conflict trials (using verbal over location specificity information) while also showing an ability to use the location specificity information when it was the only information available. This kind of selectivity has also been demonstrated with 3 - and 4-year-olds in previous research (Sophian \& Wellman, 1980).

In combination with previous studies, then, the present results portray the young preschooler as an information processer of considerable selectivity. These children are highly consistent in selecting appropriately between conflicting sources of information, and they show considerable generality in being able to extend their selectivity to new kinds of information. In essence, as early as they could be shown to make any use of a new source of information in search, they were found to be selective in handling conflict situations in which that information had to be balanced against other, competing, information about an object's location.

\section{CONCLUSIONS}

Two developmental changes are evident in early search behavior: an expansion of the range of information taken into account to guide search, and an increase in the selectivity with which children resolve conflicts between prior and current information. The development of selective information use seems to proceed through two steps. The first is a period of inconsistency in which some selectivity is evident but other patterns of responding also occur, as in the combined selectivity and perseveration of the 16-month-olds. Later, however, a more general understanding of the relative priorities among temporally ordered information is achieved, and information as to the object's most immediate position is consistently given precedence over other information. Thus, preschoolers were able to be selective even with respect to new sources of information that they were just beginning to take into account in their searches.

A strength of the present investigation was the provision of closely comparable information about the search behavior of children ranging in age from 9 months through 4 years. In fact, the results indicated considerable continuity in development at least from 16 months through 
the preschool years, an age span which has seldom been encompassed in a single investigation. The age groups shared the same basic search skills-selective search and selective information use-and differed only in the consistency and generality with which they implemented those skills. Nine-month-olds are more difficult to relate to the older groups, largely because of the lack of information pertaining to selective information use at that age. Nevertheless, the value of including them within the larger framework adopted here is apparent from the findings with respect to perseveration. While we would not want to argue from the present data that 9-month-olds never perseverate, we would take the present findings as grounds for questioning the generality of perseveration at that age. Thus, perseveration is neither unique to 9-month-olds nor especially ubiquitous in their searches. Characterizations of these infants as largely perseverative overstate the contrast between them and older infants and understate early achievements in the development of search skills. The present research indicates the utility of a broader perspective, placing perseveration within a general framework of developing search skills and patterns of information use which is applicable across a broad age range.

\section{REFERENCES}

Acredolo, L. P. Laboratory versus home: The effect of environment on the 9-month-old infant's choice of spatial reference system. Developmental Psychology, 1979, 15, 666667.

Bremner, J. G. Egocentric versus allocentric spatial coding in nine-month-old infants: Factors influencing the choice of code. Developmental Psychology, 1978, 14, 346355.

Bremner, J. G. Object localization in infancy. In M. Potegal (Ed.), The neural and developmental bases of spatial orientation. New York/London: Academic Press, 1982 (in press).

Bremner, J. G., \& Bryant, P. E. Place versus response as the basis of spatial errors made by young infants. Journal of Experimental Child Psychology, 1977, 23, 162-171.

Butterworth, G. E. Object identity in infancy: The interaction of spatial location codes in determining search errors. Child Development, 1975, 46, 866-870.

Butterworth, G. E. Asymmetrical search errors in infancy. Child Development, 1976, 47, 864-867.

Butterworth, G. E. Object disappearance and error in Piaget's Stage IV task. Journal of Experimental Child Psychology, 1977, 23, 391-401.

Evans, W. F., \& Gratch, G. The Stage IV error in Piaget's theory of object concept development: Difficulties in object conceptualization or spatial localization? Child Development, 1972, 43, 682-688.

Gratch, G. Recent studies based on Piaget's view of object concept development. In L. B. Cohen \& P. Salapatek (Eds.), Infant perception: From sensation to cognition. New York: Academic Press, 1975.

Gratch, G. Review of Piagetian infancy research: Object concept development. In W. F. Overton \& J. M. Gallagher (Eds.), Knowledge and development (Vol. 1). New York: Plenum, 1977.

Gratch, G., Appel, K. J., Evans. W. F., LeCompte, G. K.. \& Wright, N. A. Piaget's Stage IV object concept error: Evidence of forgetting or object conceptualization? Child Development, 1974, 45, 71-77. 
Gratch, G., \& Landers, W. F. Stage IV of Piaget's theory of infant object concepts: A longitudinal study. Child Development, 1971, 42, 359-372.

Harris, P. L. Development of search and object permanence during infancy. Psychological Bulletin, 1975, 82, 332-344.

Harris, P. L. The development of search. In P. Salapatek \& L. B. Cohen (Eds.), Handbook of infant perception. New York: Academic Press, 1983 (in press).

Horn, H. A., \& Myers, N. A. Memory for location and picture cues at ages two and three. Child Development, 1978, 49, 845-856.

Landers, W. F. The effect of differential experience on infants performance in a Piagetian Stage IV object-concept task. Developmental Psychology, 1971, 5, 48-54.

Loughlin, K. A., \& Daehler, M. W. The effects of distraction and added perceptual cues on the delayed reaction of very young children. Child Development, 1973, 44, 384388.

Piaget, J. The construction of reality in the child. New York: Basic Books, 1954.

Schuberth, R. E., Werner, J. S., \& Lipsitt, L. P. The Stage IV error in Piaget's theory of object concept development: A reconsideration of the spatial localization hypothesis. Child Development, 1978, 49, 744-748.

Sophian, C. Selectivity and strategy in early search. Journal of Experimental Child Psychology, 1982, 34, 342-349.

Sophian, C.. \& Wellman, H. M. Selective information use in the development of search behavior. Developmental Psychology, 1980, 16, 323-331.

Webb, R. A., Massar, D., \& Nadolny, T. Information and strategy in the young child's search for hidden objects. Child Development, 1972, 43, 91-104.

Wellman, H. M., Somerville, S. C., \& Haake, R. J. Development of search procedures in real-life spatial environments. Developmental Psychology, 1979, 5, 530-542.

\section{REFERENCE NOTES}

1. Cummings, E. M., \& Bjork, E. J. Infant search errors and the Stage IV object permanence task: Evidence for perseveration, midline preference, or forgetting? Unpublished paper.

2. Evans, W. F. The stage IV error in Piaget's theory of object concept development: An investigation of the role of activity. Unpublished doctoral dissertation, University of Houston, 1973.

ReCeIVED: February 17, 1982; REVISED: June 10, 1982. 